\title{
Hemorragia de vías digestivas altas masiva como presentación de uncinariasis
}

\section{Massive upper digestive tract hemorrhage as presentation of uncinariasis}

\author{
José Luis Fabris, Walter Chaves • Bogotá, D.C. (Colombia)
}

\section{Introducción}

La uncinariasis es una enfermedad parasitaria común en los trópicos y subtrópicos; siendo más alta en África subsahariana, seguida por Asia, América Latina y el Caribe. Las dos especies principales que causan infección en humanos son: Ancylostoma duodenale y Necator americanus $(1-2)$.

Para el 2010 se estimó que 439 millones de personas presentaban infección por uncinarias, con una prevalencia aproximada de $8 \%$, en poblaciones como Oceanía se alcanzan prevalencias hasta de $48 \%$ (3).

Los síntomas gastrointestinales son los más frecuentes y, a la vez, son inespecíficos, éstos incluyen: naúseas, diarrea, vómitos y dolor en mesogastrio; la presentación con deterioro nutricional crónico es tardía. Pese a ser lo más frecuente no son la única variedad gastrointestinal de presentación, se ha observado en reportes de casos presentación como hemorragia de intestino medio con necesidad de diagnóstico mediante cápsula endoscópica (4) e incluso con presentación como hematemesis masiva (5).

De ahí la importancia que tiene para el médico internista el conocer que la uncinariasis dentro de su espectro clínico puede manifestarse como una hemorragia de vías digestivas.

\section{Presentación del caso}

Se trata de un paciente masculino de 53 años natural y procedente de Inírida que se dedicó a construcción, agricultura y oficios varios. En el momento vivía con su esposa e hijos en una comunidad indígena, sin educación para leer ni escribir. Cursaba con un cuadro de tres días de episodios de hematemesis y deposiciones melénicas de moderada cantidad; dentro de los síntomas acompañantes se encontraban limitación para la deambulación. Tuvo su primer contacto médico en el Hospital de Boca Grande (Inírida) donde evidencian anemia con hemoglobina $(\mathrm{Hb})$ de $1.8 \mathrm{~g} / \mathrm{dL}$ por lo que trasfunden glóbulos rojos y remiten al Hospital de San José (Bogotá) por clínica compatible con hemorragia de vías digestivas altas.

Entre sus antecedentes sin evidencia de ingesta crónica de AINE, sin enfermedad ácido péptica, sin procedimientos quirúrgicos ni otros antecedentes.

A la revisión por sistemas evidenciaba dificultad para la marcha y postración en cama desde hace 10 años; adicionalmente, refería que las deposiciones melénicas habían resuelto 48 horas antes del ingreso al Hospital de San José.

Al ingreso los signos vitales eran normales, no presentaba ortostatismo, con índice de masa corporal de 19.1. Examen físico sin signos de dificultad respiratoria, auscultación cardiopulmonar sin alteraciones, sin dolor abdominal ni irritación peritoneal; tacto rectal con esfínter de buen tono, sin evidencia de hemorroides y negativo para sangrado. Al evaluar las extremidades con hipotrofia generalizada la fuerza muscular era normal, reflejos normales, sin signos de irritación meníngea, bradicinesia, con marcha de pequeños pasos, temblor en reposo y de intención. Con respecto a la piel evidenciaba palidez generalizada sin evidencia de lesiones. Los laboratorios clínicos de ingreso se encuentran en la Tabla 1.
Dr. José Luis Fabris Vargas: Residente de Medicina Interna; Dr. Walter Chaves: Especialista en Medicina Interna. Profesor Asistente. Fundación Universitaria de Ciencias de la Salud - FUCS. Bogotá, D.C. (Colombia).

Correspondencia: Dr José Luis Fabris Vargas, Bogotá, D.C. (Colombia)

E-mail: lfabris@fucsalud.edu.co 
Tabla 1. Paraclínicos iniciales.

\begin{tabular}{|c|c|}
\hline Leucocitos & $9300 \times \mathrm{mm}^{3}$ \\
\hline Neutrófilos & $8200 \times \mathrm{mm}^{3}$ \\
\hline Linfocitos & $160 \times \mathrm{mm}^{3}$ \\
\hline Eosinófilos & $300 \times \mathrm{mm}^{3}$ \\
\hline Monocitos & $300 \times \mathrm{mm}^{3}$ \\
\hline Hemoglobina & $5.9 \mathrm{gr} / \mathrm{dL}$ \\
\hline VCM & $76 \mathrm{fl}$ \\
\hline HCM & $23 \mathrm{gr} / \mathrm{dL}$ \\
\hline Plaquetas & $222000 \mathrm{x} \mathrm{mm}^{3}$ \\
\hline Reticulocitos & $8.2 \%$ (corregidos: $3.42 \%$ ) \\
\hline Bilirrubina total & $5 \mathrm{mg} / \mathrm{dL}$ \\
\hline Tiempo de protrombina & $16.9 \mathrm{seg} / 10.5 \mathrm{seg}$ \\
\hline Tiempo de tromboplastina parcial & $35.4 \mathrm{seg} / 30 \mathrm{seg}$ \\
\hline Bun & $20 \mathrm{mg} / \mathrm{dL}$ \\
\hline Creatinina & $1 \mathrm{mg} / \mathrm{dL}$ \\
\hline Aspartato amino transferasa (ASAT) & $1188 \mathrm{UI} / \mathrm{L}$ \\
\hline Alanino amino transferasa (ALAT) & $530 \mathrm{UI} / \mathrm{L}$ \\
\hline Albúmina & $2.8 \mathrm{gr} / \mathrm{dL}$ \\
\hline
\end{tabular}

En el extendido de sangre periférica se observó hipocromía, anisocitosis y esquistocitos; con serie blanca y plaquetaria normales. Se tomó perfil hepático completo con evidencia de hiperbilirrubinemia indirecta (se indagó en laboratorio acerca de dato evidenciando hiperbilirrubinemia directa con posterior normalización) y cuadro hemático con anemia microcítica hipocrómica regenerativa que obligaron a estudios carenciales y hemólisis, confirmando la primera y descartando la segunda respectivamente.

Adicionalmente, se observó elevación de enzimas hepáticas con predominio de la alanino amino transferasa (ALAT) con una relación ALAT/ASAT (aspartato amino transferasa) compatible con hepatitis aguda que hizo necesario solicitar anticuerpos para hepatotropos descartando hepatitis viral aguda y dentro de las enfermedades tropicales leptospirosis. En la Tabla 2 se anotan los paraclínicos de relevancia clínica solicitados. Con respecto a la hepatitis se indagó con familiares quienes referían consumo frecuente de medicación

Tabla 2. Paraclínicos relevancia clínica.

\begin{tabular}{|l|c|}
\hline Deshidrogenasa láctica (LDH) & $338 \mathrm{U} / \mathrm{L}$ \\
\hline Ferritina & $9.29 \mathrm{ng} / \mathrm{mL}$ \\
\hline Hierro sérico & $21 \mathrm{ug} / \mathrm{dL}$ \\
\hline Transferrina & $246 \mathrm{mg} / \mathrm{dL}$ \\
\hline Saturación de transferrina & $8 \%$ \\
\hline
\end{tabular}

naturista sin finalidad clara que nos orientó para considerar una hepatitis tóxica.

Se realizó endoscopia de vías digestivas altas con hallazgo de pliegues gástricos engrosados con placas blanquecinas en antro, sospechando Linitis plástica. No se evidenció sangrado activo ni reciente, así mismo no se evidenciaron ulceraciones.

En contexto de hallazgos endoscópicos anotados, anemia carencial e hipoalbuminemia (desnutrición) se consideró posibilidad de malignidad por lo que se iniciaron estudios de extensión que incluyeron tomografías de tórax, abdomen, cuello y cráneo (reportando atrofia cortical difusa) sin evidencia de lesiones sugestivas de neoplásica. Posteriormente, en la biopsia de lesión gástrica anteriormente descrita se documentó ulcera péptica severa.

Al descartar lo anteriormente mencionado se hizo una revisión retrospectiva del caso y se obtuvo acceso a paraclínicos extrainstitucionales donde sorpresivamente reportaban microfilarias en extendido de sangre periférica. Consideramos por ser un paciente procedente de zona rural complementar estudios con coproscópico seriado, evidenciando en estos huevos de uncinaria en las tres muestras. En vista que se habían descartado todas las otras causas de la clínica del paciente se consideró el diagnóstico de hemorragia de vías digestivas secundaria a uncinariasis por lo que se inició tratamiento con albendazol e ivermectina más reposición oral de hierro e intervención por nutrición.

El paciente egresó con los diagnósticos de hemorragia de vías digestivas altas secundaria a uncinariasis, anemia carencial secundaria, desnutrición proteicocalórica y enfermedad de Parkinson. Se hizo seguimiento un mes posterior al egreso e inicio del tratamiento con mejoría en niveles de hemoglobina y volúmenes corpusculares.

\section{Discusión}

La uncinariasis es una infección parasitaria de elevada prevalencia, se estima que hasta 4.98 millones de años de discapacidad son atribuibles a las infecciones por helmintos transmitidos por el suelo de los cuales $65 \%$ son por unicinarias (3).

El curso clínico de la infección consta de varias fases con síntomas asociados a cada una; dichas fases son: invasión larvaria dérmica (causando prurito, eritema y rash papular que comprometen principalmente manos y pies), paso transpulmonar (suele ser asintomático, en ocasiones cursa con tos leve o irritación faríngea) y paso gastrointestinal con síntomas inespecíficos (náuseas, diarrea, vómitos entre otros) que si no se corrigen progresan a insuficiencia nutricional crónica.

Al revisar este caso se hace necesario considerar la hemorragia de vías digestivas como una potencial presentación de uncinariasis y si tomamos en cuenta que no es el único caso reportado con características similares, se puede pasar de hacer un diagnóstico de exclusión a una búsqueda activa de esta entidad $(4,5)$. 
No son claros los factores que contribuyen a que se facilite la presentación como hemorragia de vías digestivas, se ha demostrado que existen factores genéticos e inmunológicos tanto del huésped como del parásito que son capaces de regular esta respuesta $(6-8)$.

Es de notar que el tratamiento de la uncinariasis que se basa en antihelmínticos no es considerado de alto costo y unas pocas dosis de albendazol, mebendazol o incluso pamoato de pirantel son suficientes para tratar la infección y lograr tasas de curación de hasta $92 \%(9,10)$. Por otro lado, la presentación de insuficiencia nutricional crónica se traduce en años de discapacidad y deterioro importante de la calidad de vida. Pese a que la insuficiencia nutricional crónica mejora con el tratamiento (11), se ha hecho necesaria la implementación de la prevención de la enfermedad para lo que se encuentran en desarrollo vacunas potenciales con resultados alentadores 12-13.

Si bien se observaron limitaciones a la hora del diagnóstico de este paciente (pobre calidad de la información, barrera de lenguaje, poca disponibilidad de historia clínica de sitio de remisión, errores en mediciones de laboratorio), no es desconocido que una adecuada anamnesis en complemento con un buen examen físico juegan un papel importante a la hora de abordar este tipo de pacientes y que muchas veces cuando no se avanza en pro del diagnóstico la estrategia más útil es devolverse y reiniciar todo el proceso, porque bien es sabido que los peores errores en medicina se basan en excelentes análisis de malas observaciones clínicas.

\section{Referencias}

1. De Silva NR, Brooker S, Hotez PJ, Montresor A, Engels D, Savioli L.. Soiltransmitted helminth infections: updating the global picture. Trends Parasitol 2003;19: 547-51.

2. Hotez PJ, Brooker S, Bethony JM, Bottazzi ME, Loukas A, Xiao S. Hookworm infection. N Engl J Med. 2004; 351: 799-807.

3. Pullan RL, Smith JL, Jasrasaria R, Brooker SJ. Global numbers of infection and disease burden of soil transmitted helminth infections in 2010. Parasit Vectors $2014 ; 7: 37$.

4. García D, Jáquez JO, González JA, Maldonado HJ. Hemorragia de intestino medio causada por uncinariasis y diagnosticada por cápsula endoscópica. Caso clínico. Rev Gastroenterol Mex. 2013; 78 (3): 196-97.

5. Montes JM, Posada JC, Cantillo K, Gómez J. Hematemesis masiva secundaria a uncinariasis: presentación de caso. Rev cienc biomed. 2016; 7 (1): 139-43.

6. Hotez PJ, Pritchard DI. Hookworm infection. Sci Am. 1995; 272 (6): 68-74.

7. Chu D, Bungiro RD, Ibanez M, Harrison LM, Campodonico E, Jones BF, et al. Molecular characterization of Ancylostoma ceylanicum Kunitz-type serine protease inhibitor: evidence for a role in hookworm-associated growth delay. Infect Immun. 2004; 72 (4): 2214-21.

8. McSorley HJ, Loukas A. The immunology of human hookworm infections. Parasite Immunol. 2010; 32 (8): 549-59.

9. Keiser J, Utzinger J. Efficacy of current drugs against soil-transmitted helminth infections: systematic review and meta-analysis. JAMA. 2008; 299 (16): 1937-48.

10. Seinmann P, Utzinger J, Du ZW, Jiang JY, Chen JX, Hattendorf J, et al. Efficacy of single-dose and triple-dose albendazole and mebendazole against soil-transmitted helminths and Taenia spp.: a randomized controlled trial. PLoS One. 2011; 6 (9): e25003.

11. Bethony J, Brooker S, Albonico M, Geiger SM, Loukas A, Diemert D, et al. Soil-transmitted helminth infections: ascariasis, trichuriasis, and hookworm. Lancet. 2006; 367 (9521): 1521-32

12. Diemert DJ, Bethony JM, Hotez PJ. Hookworm Vaccines. Clin Infect Dis. 2008; 46 (2): 282-8

13. Bethony JM, Simon G, Diemert DJ, Parenti D, Desrosiers A, Schuck S, et al Randomized, placebo-controlled, double-blind trial of the Na-ASP-2 hookworm vaccine in unexposed adults. Vaccine. 2008; 26 (19): 2408-17. 